\title{
New FEM - Programs for Modeling Coupled Processes of Selective Vapor Condensation during Laser Processing of Materials
}

\author{
Igor N. Sachkov ${ }^{1, a)}$, Viktoria F. Turygina ${ }^{1, \text { b) }}$, Vitaly Ford ${ }^{2}$ c), Dmitry G. Ermakov ${ }^{1)}$ \\ ${ }^{1}$ Ural Federal University, Mira Street, 19, Yekaterinburg, Russia, 620002 \\ ${ }^{2}$ Arcadia University, 450 S. Easton Road, Glenside, PA 19038, USA \\ a)insatch@e1.ru \\ ${ }^{\text {b)} C o r r e s p o n d i n g ~ a u t h o r: ~ v . f . v o l o d i n a @ u r f u . r u ~}$ \\ c)fordv@arcadia.edu
}

\begin{abstract}
This research presents a computer model for conjugate heat and mass transfer during laser processing of materials. The computer programs based on FEM, which allow to predict the processes of formation of evaporation and condensation zones on the surface areas surrounding the crater are implemented. There is the presence of three modes of operation, differing in the configuration of evaporation zones - condensation. The identified control parameters change modes. A phase diagram for the implementation of process modes is proposed. Finally, the research discusses the possibility of managing the process.

Keywords: laser technology, finite element method, coupled processes, evaporation, condensation, diffusion interaction.
\end{abstract}

\section{INTRODUCTION}

One of the least-studied features of laser processing of materials is the formation of condensation raids.

The purpose of this work is the development of computational methods that make it possible to describe the processes of evaporation-condensation on the working surfaces of the materials being processed during laser irradiation.

There are a number of computer approaches to the description of the processes of heat and mass transfer in the processes of cutting, welding, spraying and synthesis of powders, but the patterns of condensation processes for the surface area adjacent to the heating zone are not well understood.

Let us consider the conjugate processes of heating the material, evaporation of its components, diffusion of vapors in the background gas and possible condensation. It is necessary to find out under what conditions the discussed condensation occurs, and under what conditions it does not.

\section{FORMALISM OF THE DEVELOPED METHOD}

We will consider the situation in which the beam heats a portion of the target surface, forms a temperature field on the surface and in the depth of the target, and eventually creates a stream of sublimation. The scheme of the configuration under consideration is shown in Fig. 1a. Figure 1 shows the region of heating of the sample by a laser (crater). As a result of heating the material, the temperature distribution $T(R, Z)$ is formed in it, characterized by axial symmetry, and in the surrounding gas - the distribution $\mathrm{P}(\mathrm{R}, \mathrm{Y})$ of the partial pressure of the material vapor. We take into account that not only the crater can serve as a source of vapors, but also heated sections of the sample adjacent to the crater. In this case, a diffusion interaction is created between the surface of the material and the surrounding gas, which determines whether evaporation or condensation will take place.

International Conference of Numerical Analysis and Applied Mathematics ICNAAM 2019

AIP Conf. Proc. 2293, 120018-1-120018-4; https://doi.org/10.1063/5.0026680

Published by AIP Publishing. 978-0-7354-4025-8/\$30.00 
We take into account that the temperature distribution in the sample volume is determined by the action of heat sources uniformly distributed over the surface of the crater and the distribution of heat in the sample volume (region "b" in Fig. 1). Under the conditions considered, in the center of the crater of a beam (crater), the temperature of the target surface, Tmax, is maximal and then monotonously decreases with distance from the zone of action of the beam, tending to a fixed value To.

In this paper, we study the situations that occur when there is a background gas environment that does not interact chemically with the migrating pairs. Under the conditions discussed, the pairs are able to diffuse in the working volume (region "c" in Fig. 1) and then either leave the working region or partially condense in colder areas of the target surface.

To solve the discussed related problems of heat transfer, vapor diffusion and evaporation-condensation processes, in this work, new programs based on the Finite Element Method (FEM) were developed. At the same time computer technologies were developed, proposed in a number of previous works. In them, FEM was used to analyze synergistic processes during the evaporation of substances from porous materials [1], prediction of condensation patterns of volatile fission products [8], analysis of failures of electronic vacuum devices [3], patterns of vapor absorption in porous materials [4], precision layers of microelectronics products [5].

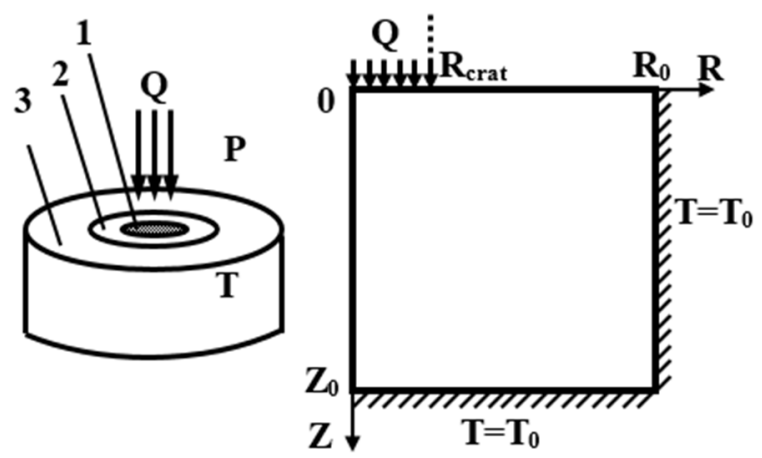

$\mathbf{b}$

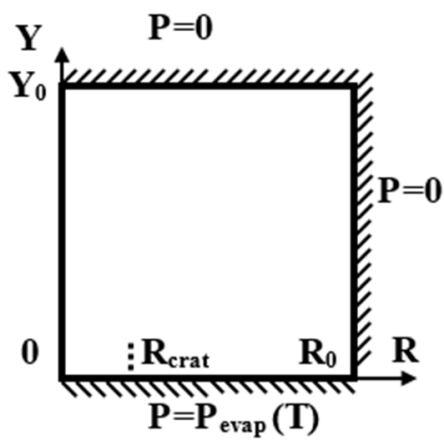

FIGURE 1. Configurations of heat and mass transfer processes under laser exposure; a - process diagram; b, c - working areas of heat transfer and diffusion, respectively. Arrows and hatching - the scope of the boundary conditions of the second and first kind, respectively.

In contrast to the works cited above, in this study, the temperature field is not arbitrarily set, but calculated. At the same time, the main program organizes the work of two subprograms that calculate temperatures and calculate evaporation-condensation flows. The "thermal" design area is shown in Fig. 1b. A cylindrical coordinate system is used, the beginning of which is aligned with the center of the crater. $\mathrm{Z}$ axis is directed deep into the material. The dimensions of the computational domain $\mathrm{R} 0, \mathrm{Z} 0$ were chosen from the condition of the accuracy of calculations equal to $2 \%$. Rcrat is the crater radius. The following boundary conditions were taken into account. In the area of the crater, $Z=0, R c r a t \geq R \geq 0$, the conditions of the 2 nd kind, that is, the presence of heat flux density $Q$. At the boundaries $\mathrm{R}=\mathrm{R} 0$ and $\mathrm{Z}=\mathrm{Z} 0$, highlighted in Fig. $1 \mathrm{~b}$ by shading, conditions of the 1 st kind are satisfied, $\mathrm{T}=\mathrm{T} 0$.

The calculated temperature distribution over the sample surface was placed in an array, which was then used to calculate the vapor pressure distribution and the densities of the evaporation and condensation flows. A diagram of the vapor distribution area is presented in Fig. 1c.

A cylindrical coordinate system is used, the beginning of which is aligned with the center of the crater. The Y axis is directed deep into the gas region. The dimensions of the computational domain Y0 were chosen from the condition of the accuracy of calculations equal to $2 \%$. The following boundary conditions were taken into account. At the boundaries $\mathrm{R}=\mathrm{R} 0$ and $\mathrm{Y}=\mathrm{Y} 0$, the conditions of the 1 st kind, $\mathrm{P}=0$, which means the dispersion of vapors in the surrounding background gas.

At the boundary $\mathrm{Y}=0$, corresponding to the surface of the sample, the vapor pressure and saturation pressure, Pev, is calculated at the temperature of this surface point Pev = Bo exp (- Qev / Rg T), in accordance with the Clapeyron dependence. Qev is the heat of evaporation of the material, $\mathrm{Rg}$ is the gas constant, Bo is an insignificant large-scale factor.

To find the vapor pressure distribution $\mathrm{P}(\mathrm{R}, \mathrm{Y})$, the principle of externality of the functional was used [7]: 


$$
\chi_{P}=\int_{V_{P}} D(\operatorname{grad} P)^{2} d V
$$

where $\mathrm{D}$ is the vapor diffusion coefficient, which is assumed to be weakly dependent on the gas temperature and therefore represents an insignificant scale factor, VP is the volume of the calculated gas region.

Further, the standard FEM scheme was used [1]. Its use led to the establishment of the distribution of vapor pressure $\mathrm{P}(\mathrm{R}, \mathrm{Y})$ and then to the calculation of the flux densities of their evaporation-condensation on the sample surface $\mathrm{q}=\mathrm{D}(\partial \mathrm{C} / \mathrm{Y}) \mathrm{Y}=0$, while the positive sign of $\mathrm{q}$ corresponded to evaporation, and negative - condensation.

\section{RESULTS OF CALCULATIONS}

The above program was used to conduct computational experiments in which the effect of thermal and geometric features of the irradiation process on the formation of evaporation and condensation zones was studied. As a rule, partitioning grids with a density of 200x 200 elements were used. The errors in calculating the values of temperature and concentration were estimated using the methods described in the works cited above [2-5]. In this case, only those results were taken into account, which were characterized by a relative error not exceeding $2 \%$.

Figure 2 presents the characteristic dependences of the relative flux density of evaporation-condensation on the relative distance from the center of the crater. On it $\mathrm{q}$ is the mass flux density on the sample surface, if $\mathrm{q}>0$, evaporation occurs, if $\mathrm{q}<0$ is condensation. $\mathrm{q} 0$ is the evaporation flow modulus in the center of the crater.

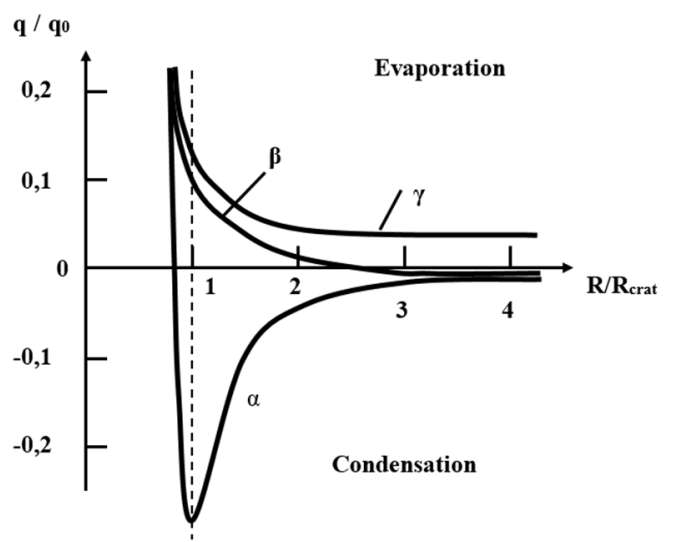

FIGURE 2. Radial dependences of evaporation and condensation flux densities from the target surface, calculated with different irradiation parameters, Tmax / T0 and Q / (Rg Tmax); they are equal respectively: " $\alpha$ " - 10 and 2; " $\beta "$ - 1.5 and $0.7 ; " \gamma "-1.5$ and 0.07 .

The figure illustrates the presence of three qualitatively different situations, "modes" of the process. In the case of " $\gamma$ ", evaporation takes place over the entire surface of the target. The vapors do not condense on it, but only diffuse into the volume of the background gas. The " $\alpha$ " curve reflects the situation when vapor condensation occurs over the entire surface of the sample, including part of the crater. The " $\beta$ " situation is intermediate between $\alpha$ and $\gamma$, that is, evaporation occurs on the part of the target adjacent to the crater, and condensation occurs on the remaining colder one. The situations described are similar to those found earlier in the works cited [1-6], which are devoted to thermal evaporation-condensation processes in various objects.

\section{DISCUSSION}

Systematization of the results of the computational experiments made it possible to present the conditions for the formation of $\alpha, \beta$ and $\gamma$ modes in the form of a diagram presented in Fig. 3. On it, Tmax is the maximum temperature realized in the center of the crater, T0 is the minimum (initial) temperature of the material. Q is the specific heat of evaporation of the material, $\mathrm{Rg}$ is the gas constant.

In this case, the control parameter (Tmax / T0) implicitly characterizes the heat input $\mathrm{q}$ and the thermal conductivity coefficient of the substrate $\lambda$. The control parameter Q / (Rg T0) is determined by the volatility of the vapor. Analyzing the type of diagram, we can conclude that the control parameters allow us to vary the course of the evaporation process - condensation during laser processing. In particular, if the set of parameters corresponds to 
the point "c" in Fig. 3 (low radiation power, low vapor volatility), then vapor does not settle on the sample surface. If we increase the radiation power (point "a"), then condensation will occur over the entire surface. An intermediate mode corresponding to " $b$ " causes the crater to surround the ring of evaporation, beyond which condensation takes place.

If the material is an alloy of two components, which differ significantly in the heat of evaporation, then coexistence of situations "c" and " $\mathrm{d}$ " is possible. In this case, the more volatile component is dispersed in the background gas, and the less volatile (point "d") is deposited on the surface. Thus, the separation of alloy components is possible.

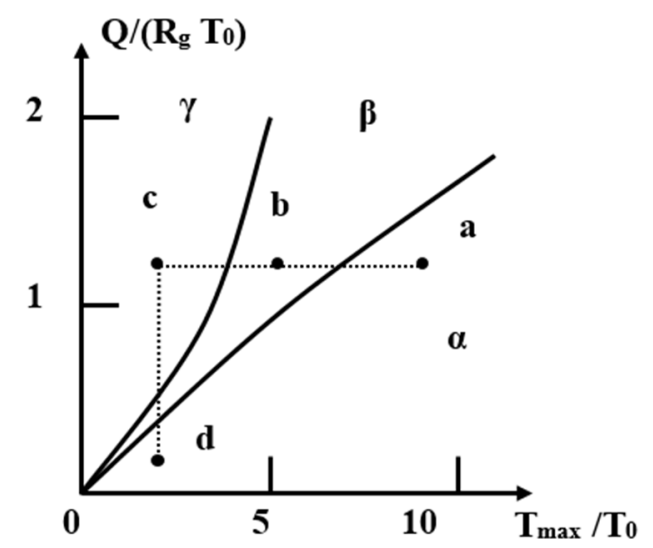

FIGURE 3. Stability diagram of various modes of evaporation-condensation processes during laser processing of materials.

It should be noted that the computational method presented above can be used further to describe the processes of evaporation-condensation of water and salts, recently recorded on the surfaces of asteroids [8]. In the discussed objects at the borders of the zones illuminated by the sun and in the shade, the processes of water vapor migration and the occurrence of ice raids are possible.

\section{CONCLUSIONS}

When a laser is applied to a target, diffusion interaction processes of the heated surface and vapors are formed, which leads to the formation of evaporation and condensation zones. A computational-based method for predicting processes is presented. Results can be used to optimize laser processing. In the future, it is planned to develop an approach that allows taking into account a number of additional factors: the shape, thickness and configuration of the sample, the non-stationary irradiation and others.

\section{REFERENCES}

[1] Sachkov, I.N., Turygina, V.F., Medvedev, M.A., Dolganov, A.N., \& Spasov, K.B., "Development of new FEM programs for synergistic evaporation processes from porous systems" AIP Conference Proceedings 2040, 050017 (2018).

[2] I.N. Sachkov, High Temperature 33, № 5, 759 (1995).

[3] I.N. Sachkov and P.V Gel’d, Technical Physics Letters 19, № 20 (1993).

[4] I.N. Sachkov, Russian Journal of Physical Chemistry 70, № 1, 111 (1996).

[5] P.V Gel’d and I.N. Sachkov, Doklady Physics 325, № 2 (1992).

[6] Igor N. Sachkov, Dmitry B. Berg, Aleksey G. Gorman, Victoria F. Turygina and Oleg E. Khorev, "Optimization of the characteristics of three-phase resistive materials using finite element method", AIP Conference Proceedings 1863, 050012 (2017); https://doi.org/10.1063/1.4992209.

[7] L. Segerlind, Applied Finite Element Analysis, 2-nd edition (Wiley, New York, 1984).

[8] A. Nathues, M. Hoffmann J.-B., "Vincent Sublimation in bright spots on (1) Ceres", Nature, Vol. 528, pp. 237-240 (10 December 2015). 